\title{
Effektivität der Manuellen Lymphdrainage in der Erhaltungsphase einseitiger, sekundärer Armlymphödeme - Eine Pilotstudie*
}

\author{
Efficacy of Manual Lymphatic Drainage in the Maintenance-Phase Treatment \\ of Unilateral, Secondary Arm Lymphedema - A Pilot Study
}

Autoren

Institute
C. Daubert ${ }^{1}$, J. Rude ${ }^{2}$, W. Schobersberger ${ }^{3}$, G. Hoffmann ${ }^{3}$

Berufsförderungswerk Mainz

Institut für Funktionelle und Klinische Anatomie, Universität Mainz

Department für Medizinische Wissenschaften und Management, UMIT - Private Universität für Gesundheitswissenschaften, Medizinische Informatik und Technik

\section{Bibliografie}

Dol http://dx.doi.org/

10.1055/s-0030-1256091

Akt Dermatol 2011; 37:

114-118 @ Georg Thieme

Verlag KG Stuttgart · New York

ISSN 0340-2541

Korrespondenzadresse

Constance Daubert

Fachlehrerin für Komplexe Physikalische

Entstauungstherapie

Diplom-Physiotherapeutin

Berufsförderungswerk Mainz

Lortzingstr. 4

55127 Mainz

Conny-Daubert@gmx.de

\section{Zusammenfassung}

$\nabla$

Bei einer durchschnittlichen jährlichen Lymphödeminzidenzrate von rund 16200 betroffenen Frauen und Männern nach einer Mammakarzinombehandlung liegen derzeit nur elf hochwertige Studien zum Thema einer effektiven Kurzbzw. Langzeitbehandlung vor. Diese Thematik bearbeitende, aktuelle Metaanalysen weisen einen klaren Mangel im Bereich der Evidenzbasierung der Manuellen Lymphdrainage (MLD) auf. Ziel dieser Studie war es, die Effektivität der Manuellen Lymphdrainage in der Erhaltungsphase der Komplexen Physikalischen Entstauungstherapie (KPE) über einen Zeitraum von drei Monaten zu prüfen. 76 Patientinnen wurden in eine von vier Behandlungsgruppen (Vergleich MLD, KPE, Kom- pressionstrumpf und Kinesio-Taping Technik [KTT]) randomisiert. Die Datengewinnung erfolgte in vier Messungen. Die Varianzanalyse stellte im Vergleich der vier Gruppen einen signifikanten Unterschied von 0,042 ( $\mathrm{p}<0,05)$ fest. Die Prüfung der Variablen innerhalb der vier Gruppen (Friedman-Test) ergab in der Kompressionsstrumpfgruppe eine signifikante Reduktion $(0,001)$. Keine signifikanten Unterschiede konnten im prä-post-Vergleich (Friedman-Test) in den Gruppen MLD, KTT und KPE festgestellt werden. Die Ergebnisse der Gruppen KPE, MLD und KTT weisen aufgrund eines fehlenden signifikanten Unterschieds eine mögliche Gleichwertigkeit auf. Die Kompressionsbestrumpfung zeigt eine signifikante Überlegenheit.

\section{Einleitung}

$\checkmark$

Sekundäre Lymphödeme sind eine mögliche Komplikation nach notwendigen Operationen und Bestrahlungen bei Brustkrebserkrankungen. Diverse Studien geben Lymphödeminzidenzen zwischen $6-46,3 \%$ an [1-7]. Aktuellere Studien grenzen die Inzidenz zwischen 8,4 und $33 \%$ ein [8-10]. Trotz einer jährlichen Brustkrebsneuerkrankungsrate in Deutschland von ca. 60000 Frauen [11] und somit (bei einer durchschnittlichen Inzidenzrate von 20,7) einer Rate von rund 16200 Lymphödemneuerkrankungen pro Jahr liegen derzeit nur elf hochwertige Studien [1222] zum Thema einer effektiven Kurz- bzw. Langzeitbehandlung sekundärer Lymphödeme vor. Von diesen untersucht lediglich die Studie von Andersen et al. (2000) [23] die Erhaltungsphase (Phase 2) der Komplexen Physikalischen Entstauungstherapie (KPE). Die Erhaltungsphase umfasst

\footnotetext{
* Finanzielle Unterstützung der Studie durch das Berufsförderungswerk Mainz.
}

Manuelle Lymphdrainage, das Tragen eines Kompressionsstrumpfes, selbstdurchgeführte Bewegungsübungen und Hautpflege mit dem Zweck des Ödemstatuserhalts. Diese Phase folgt einer vier- bis sechswöchigen Entstauungsphase (Phase 1) des Lymphödems. Die mit dem Ziel der Evidenzbasierung der KPE und deren einzelnen Komponenten (Kompression; Manuelle Lymphdrainage; Hautpflege; Bewegung in Kompression) durchgeführten Studien erreichen aufgrund „fehlerhafter Studiendesigns und kleiner Fallzahlen“ nur eine begrenzte Aussagefähigkeit [24]. Neben der Metaanalyse von Beckermann zeigen auch die Metaanalyse von Schiller - Frühwirt (2007) [25] und ein Review von Devoogt et al. (2010) [26] einen klaren Mangel im Bereich des Effektivitätsnachweises der Manuellen Lymphdrainage auf. Im Gegensatz dazu besteht eine eindeutige Validisierung der Effektivität für die KPE (Kompression; Manuelle Lymphdrainage; Hautpflege; Bewegung in Kompression). Hinsichtlich der vier einzelnen Behandlungskomponenten der KPE konnten bisher die Kompression bezüglich einer 
Ödemreduktion [27-31] und die Bewegungsübungen (in Kompression) bezüglich einer Schmerzreduktion und einer allgemeinen Gesundheitsverbesserung [32-36] validiert werden. Belege, dass Bewegungsübungen zu einer signifikanten Reduktion des Ödemvolumens führen, liegen derzeit nicht vor. Der Mangel in der Evidenzbasierung der Manuellen Lymphdrainage und die RCT von Andersen et al. 2000 [37], in der die Manuelle Lymphdrainage in der Phase 2 der KPE keinen zusätzlichen Effekt aufwies, führten bei Beckermann (2008) [38] zu der Fragestellung, ob die Therapie sekundärer Lymphödeme mittels der MLD in der Erhaltungsphase als Fehltherapie zu erachten ist. Dieses Problem aufgreifend, war das Ziel dieser Pilotstudie mit prospektivem, randomisiertem Design, die Effektivität der Manuellen Lymphdrainage in der Erhaltungsphase der KPE an einseitig betroffenen Lymphödemerkrankten nach Mammakarzinom zu prüfen. Die Prüfung der Effektivität wurde im direkten Vergleich zur KPE, zum alleinigen Kompressionsstrumpf und zur neueren Kinesio-Taping-Technik (Lymphtaping) durchgeführt.

\section{Methode \\ $\nabla$}

In diese multizentrische Pilotstudie, welche zuvor von drei Ethikkommissionen (Rheinland-Pfalz, Hessen, Universitätsklinikum Giessen) freigegeben wurde, wurden 76 Patientinnen im Alter von durchschnittlich 62,9 Jahren (Altersbereich: 30-75) aufgenommen. Die Rekrutierung der Patientinnen erfolgte über die Deutsche Lymphselbsthilfe. Insgesamt wurden sechs Einschlusskriterien festgelegt ( $\bullet$ Abb. 1).

Aus der Studie ausgeschlossen wurden Patientinnen im Finalstadium, mit Auftritt von Rezidiven, akuten Erysipelinfekten, kardialen Grunderkrankungen und Patientinnen, die eine Range of motion (ROM) unter 90-Grad-Flexion und Abduktion aufwiesen. Die ROM wurde im Hinblick auf eine funktionierende, nach Kase et al. (2006) [39] für die Kinesio-Taping-Technik relevante, Muskelpumpentätigkeit festgelegt.

\section{Randomisierung und Studiendesign}

Die Patientinnen wurden für drei Monate in eine von vier möglichen Behandlungsgruppen randomisiert. Die Randomisierung erfolgte extern. Die Behandlung der ersten Gruppe (KPE-Kontrollgruppe) bestand aus dem Tragen einer Kompressionsbestrumpfung (nicht älter als sechs Monate) und 2-mal wöchentlicher Manueller Lymphdrainage ( $45 \mathrm{~min}$.). Die zweite Gruppe erhielt nur Manuelle Lymphdrainage (2-mal wöchentlich/45 min.). Die dritte Gruppe trug einen Kompressionsstrumpf (durchschnittliche Tragedauer 8,2 Stunden/d [Bereich: 6,7-10,75 Stunden]). In der vierten Gruppe wurden die Teilnehmerinnen mittels der Kinesio-Taping-Technik behandelt. Alle 76 Patientinnen führten zweimal wöchentlich ein festgelegtes, während des Aufklärungsgesprächs besprochenes und schriftlich fixiertes Bewegungsprogramm sowie die Hautpflege selbstständig durch.

\section{Störvariablen}

Sechs mögliche Störvariablen wurden identifiziert, welche durch Gegenmaßnahmen reduziert bzw. unterbunden wurden:

a) Unterschiede in der Behandlung der Patientinnen aufgrund unterschiedlicher Fortbildungskonzepte im Bereich der Manuellen Lymphdrainage/KPE

b) Unterschiede in der Behandlung der Patientinnen aufgrund unterschiedlicher Fortbildungskonzepte im Bereich des Kinesio-Taping-Konzeptes (Lymphtaping)
Benignes, sekundäres, einseitiges Armlymphödem

\begin{tabular}{|c|c|c|c|}
\hline $\begin{array}{l}\text { Umfangzunahme des } \\
\text { betroffenen Arms im } \\
\text { Seitenvergleich zur } \\
\text { gesunden Seite von } \\
\text { mindestens } 2 \mathrm{~cm} \\
\text { an zwei aufeinander- } \\
\text { folgenden Mess- } \\
\text { punkten (Schüne- } \\
\text { mann-Kriterium) }\end{array}$ & \multicolumn{2}{|c|}{$\begin{array}{l}\text { Teilnehmerinnen in } \\
\text { der Erhaltungsphase } \\
\text { (Phase 2) der KPE }\end{array}$} & $\begin{array}{l}\text { Gute Compliance } \\
\text { bezüglich der } \\
\text { Kompression, } \\
\text { Bewegungsübungen } \\
\text { und der selbstständig } \\
\text { durchgeführten } \\
\text { Hautpflege }\end{array}$ \\
\hline \multicolumn{2}{|c|}{$\begin{array}{l}\text { Ärztliches Aufklärungsgespräch } \\
\text { und unterschriebene } \\
\text { Einverständniserklärung }\end{array}$} & \multicolumn{2}{|c|}{$\begin{array}{l}\text { Beginn sechs Monate nach der } \\
\text { Chemotherapie aufgrund von } \\
\text { Taxanen }\end{array}$} \\
\hline
\end{tabular}

Abb. 1 Einschlusskriterien.

c) Die Compliance der Patientinnen

d) Kompressionsdruck und Sitz des Strumpfes

e) Durchführung der Eingangs- und Endmessung

f) Überprüfung der monatlichen Dateneingabe in Case Report Forms.

Unterschiede hinsichtlich der Manuellen Lymphdrainage - und der Kinesio-Taping-Fortbildung (Differenzen im praktischen Aufbau der Behandlungen) wurden durch eine Schulung der Therapeuten aufgehoben. Griffreihenfolge und Druckstärke wurden kontrolliert (handelsübliche Druckmanschette zur Druckstärkenkontrolle) und schriftlich fixiert. Sowohl die Manuelle Lymphdrainage als auch die Kinesio-Taping-Technik folgten einer genau festgelegten Reihenfolge. Die Applikation der Streifen fand in maximaler Hautdehnung ohne Zug (0\%) auf das Tape statt. Die Schulung führte zu einer einheitlichen Durchführung sowohl der Manuellen Lymphdrainage als auch der Kinesio-Taping-Einheiten. Die Compliance der Patientinnen wurde mittels Patientinnentagebüchern geprüft. Entscheidend waren in diesem Zusammenhang Aufklärungsgespräche, die die Dringlichkeit der Compliance deutlich aufwiesen. Auch der Kompressionsdruck, der Sitz und das Alter (maximal 6 Monate) des Kompressionsstrumpfs wurden zu Beginn der Studie einer Prüfung unterzogen (eine Untersucherin). Die Durchführung der Behandlungen, die Datenerfassung in Case Report Forms (CRFs) und die Compliance der Teilnehmerinnen wurden in zuvor extern randomisierten Kontrollbesuchen überprüft. Diese Kontrolle erfolgte bei $25 \%$ der Teilnehmer. Die Datensätze wurden in insgesamt vier Messungen (Erstbefund [U0], Messung nach einem Monat [U1], Messung nach zwei Monaten [U2] und Endbefund [U3]) nach der $4 \mathrm{~cm}$-Methode [40] erhoben. Zur Objektivierung der Datensätze wurden die Umfangsmessungen U0 und U3 von derselben Therapeutin durchgeführt [41]. Vor der Durchführung der U3 hatte die Therapeutin nicht die Möglichkeit der Einsicht in die bei der Eingangsmessung gewonnen Daten. Die von den teilnehmenden Therapeuten durchgeführte U1- und U2-Messungen dienten der Verlaufskontrolle. In diesem Zusammenhang wird darauf hingewiesen, dass laut Seifart et al. (2010) [42] kein signifikanter Unterschied bei Umfangmessungen mit standardisiertem Messband, welche bei einer Patientin von mehreren messenden Therapeuten durchgeführt werden, besteht. 


\section{Ergebnisse mit statistischer Analyse \\ $\nabla$}

Nach Beendigung der praktischen Studienphase konnten 60 Datensätze ausgewertet werden. In 16 Fällen kam es zum Abbruch der Studie ( $\bullet$ Abb. 2).

Die von November 2008 bis Mai 2010 erhobenen Datensätze wurden mittels des Statistikprogramms IBM ${ }^{\circledR}$ SPSS $^{\circledR}$ Statistics 18 ausgewertet. Die im Zuge der ANOVA-errechneten Mittelwerte der Ödemvolumina ( $\triangle$ Abb.3) in den vier Gruppen weisen auf, dass das Tragen des Kompressionsstrumpfs mit - $177 \mathrm{ml}$ (SD 201,99) Volumenreduktion die höchste Effektivität aufweist.

Die KPE als Kombinationstechnik folgt mit - 38,60 ml (SD 164,53) Reduktion. Die KTT und die Behandlung mittels der Manuellen Lymphdrainage weisen Volumenzunahmen mit 5,23 ml (SD 250,63) und 13,43 $\mathrm{ml}$ (SD 175,98) auf. Ein signifikanter Unterschied im Vergleich der Gruppen konnte mit $\mathrm{p}<0,042$ zugunsten der Kompression festgestellt werden. Die Prüfung der Variablen innerhalb der vier Gruppen (Friedman-Test) ergab in der Kompressionsstrumpfgruppe eine signifikante Reduktion $(\mathrm{p}<0,001)$. Kein signifikanter Unterschied konnte im prä-post-Vergleich (Friedman-Test) in den Gruppen MLD, KTT und KPE festgestellt werden.

\section{Diskussion und Schlussfolgerungen \\ $\nabla$}

Die Manuelle Lymphdrainage weist bezüglich des Ödemvolumens mit $+13,43 \mathrm{ml}$ eine Ödemvolumenzunahme auf und unterstützt somit die von Andersen et al. (2000) publizierten Werte. Die hohen Standardabweichungen der Studie von 210,5 ml (gesamt) decken sich mit den Standardabweichungen des Papers von Andersen et al. 2000 (SD 209,3 ml) [43] und liegen unter den Standardabweichungen von Meijer et al. (2004) [44] mit Werten zwischen 606-467,3 ml und den Standardabweichungen von Karges et al. (2003) [45] mit 613,5 ml. In diesem Zusammenhang sollten der unterschiedliche Status der fibrosklerotischen Veränderungen der jeweiligen Patientinnen zu Beginn der Studie sowie die geringe Probandenzahl $(n=76)$ bedacht werden. Die während der U0 und der U3 erfolgten Inspektionsund Palpationsbefunde (eine Untersucherin) geben einen Hinweis darauf, dass in den Gruppen MLD, KTT und KPE der Status der fibrosklerotischen Veränderungen verbessert werden konnte. Eine Erklärung für diese Ergebnisse könnte die durch die MLD und durch die KTT begünstigte Thixotropie sein, bei der es zu „Veränderungen kolloidaler Systeme aus dem Gel- in den Solzustand, aufgrund mechanischer Kräfte kommt“ [46]. Die MLD und die KTT könnten somit über den verbesserten Abbau von Substanzen entzündungsreduzierend wirken, was im Bereich der MLD bezüglich der Oxysterole (entzündungsunterstützende Wirkung) nachgewiesen scheint [47]. Davon abweichend konnte eine Verschlechterungen (Verhärtung und Vergrößerung) der fibrosklerotischen Veränderungen bei alleiniger Kompressionsbestrumpfung beobachtet werden. Leduc et al. (1985) [48] und Partsch et al. (1981) [49] unterstützen in ihren Publikationen diese Beobachtung, indem sie darauf hinweisen, dass Drucktherapie keine Aufnahme von Proteinen in das Lymphgefäßsystem begünstigt. Leduc et al. ergänzten 1997 [50], dass Druck lediglich den Abtransport bereits reabsorbierter Proteine unterstütze. Dies fördert die Annahme, dass die MLD zwar im Bereich der Ödemvolumenreduktion schlechte Langzeitdaten, jedoch im Bereich der Lösung fibrosklerotischer Veränderungen gute Werte aufweist. Der Aussage von Beckermann (2008) [38], dass die

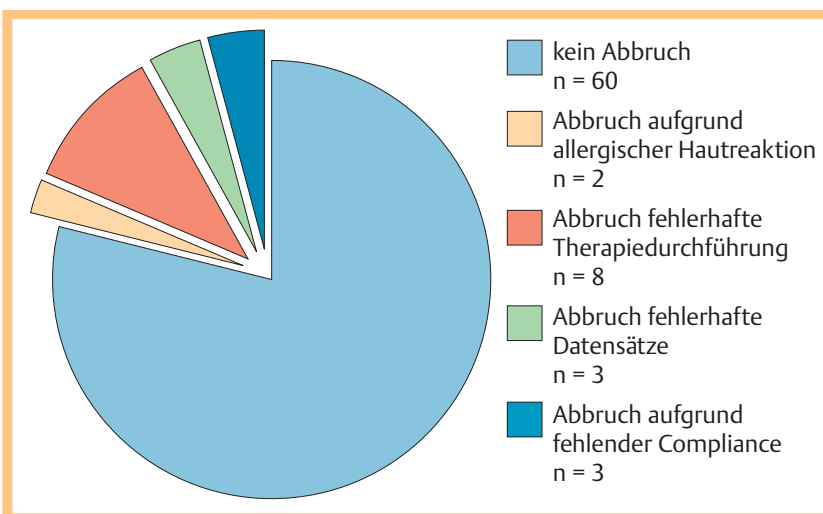

Abb. 2 Studienabbruch; Gründe und Teilnehmeranzahl. Grundgesamtheit $\mathrm{n}=76$.

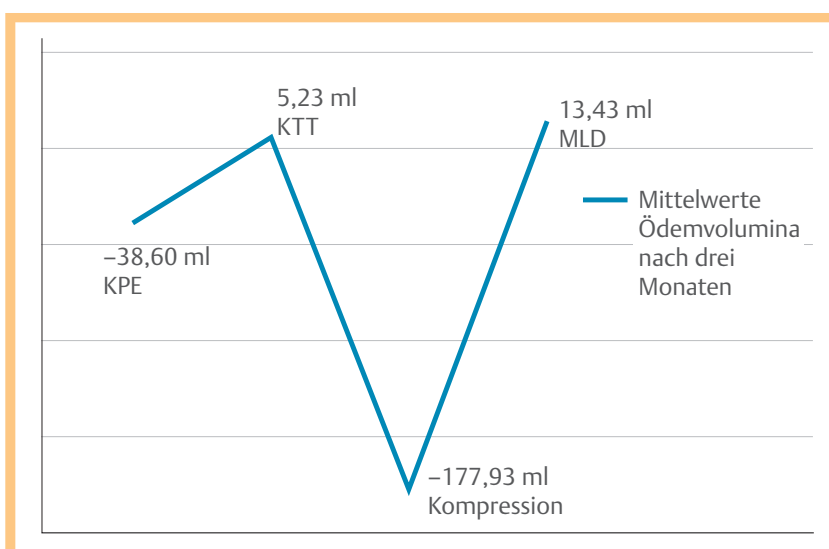

Abb. 3 Ödemvolumina der vier Behandlungsgruppen nach drei Monaten. $\mathrm{KPE}=$ Komplexe Physikalische Entstauungstherapie; KTT = Kinesio-TapingTechnik; MLD = Manuelle Lymphdrainage.

MLD eine „Fehltherapie“ sei, kann somit nur bedingt zugestimmt werden. Weitere Studien werden benötigt, um einen gezielteren Einsatz der MLD in der Komplexen Physikalischen Entstauungstherapie definieren zu können.

\section{Abstract}

\section{Efficacy of Manual Lymphatic Drainage in the Maintenance-Phase Treatment of Unilateral, Secondary Arm Lymphedema - A Pilot Study} $\nabla$

The average annual incidence of lymphedema following breast carcinoma treatment is approximately 16.200 cases in women and men, however only eleven studies of high quality on effective short-term or long-term therapy are currently available. Recent meta-analyses which address this topic are clearly deficient with regard to the evidence base for manual lymphatic drainage. The aim of this study was to review the efficacy of manual lymphatic drainage (MLD) in maintenance-phase complex decongestive physiotherapy (CDP) over a period of three months. 76 female patients were randomly assigned to one of four treatment groups (comparison of MLD, CDP, compression sleeve and Kinesio Taping (KT) method). The data were acquired in four measurements. The analysis of variance revealed a significant difference of $0,042(p<0,05)$ in the comparison of the four groups. 
A review of the variables within the four groups (Friedman test) showed a significant reduction $(0,001)$ in the compression sleeve group. No significant differences were found in the pre-post comparison (Friedman test) in the MLD, KT und CDP group. In light of the absence of a significant difference, the results of the CDP, MLD and KT groups indicate a potential equivalence. The compression sleeve group showed significant superiority.

\section{Literatur}

1 Deevogdt N, Van Kampen M, Geraerts I et al. Different physical treatment modalities for lymphoedema developing after axillary lymph node dissection for breast cancer: a review. Eur J Obstet Gynecol Reprod Biol 2010; 149: 3-9

2 Hayes SC, Reul-Hirche H, Turner J. Exercise and secondary lymphedema: safety, potential benefits, and research issues. Med Sci Sports Exerc 2009; 41: 483-489

3 Netopil B. Lymphödem bei Mammakarzinom. Frauenarzt 2008; 49: $322-325$

4 Deutsch M, Land S, Begovic $M$ et al. The incidence of arm edema in women with breast cancer randomized on the National Surgical Adjuvant Breast and Bowel Project study B-04 to radical mastectomy versus total mastectomy and radiotherapy versus total mastectomy alone. Int J Radiat Oncol Biol Phys 2008; 70: 1020-1024

5 Campisi C, Davini D, Bellini C et al. Is there a role for microsurgery in the prevention of arm lymphedema secondary to breast cancer treatment? Microsurgery 2006; 26: $70-72$

6 Mansel RE, Fallowfield L, Kissin $M$ et al. Randomized multicenter trial of sentinel node biopsy versus standard axillary treatment in operable breast cancer: the ALMANAC trial. JNCI 2006; 98: 599-609

7 Petrek JA, Heelan MC. Incidence of breast carcinoma-related lymphedema. Cancer 1998; 83 (12 Suppl. American): 2776-2781

8 Deevogdt N, Van Kampen M, Geraerts I et al. Different physical treatment modalities for lymphoedema developing after axillary lymph node dissection for breast cancer: a review. Eur J Obstet Gynecol Reprod Biol 2010; 149: 3-9

9 Hayes SC, Reul-Hirche H, Turner J. Exercise and secondary lymphedema: safety, potential benefits, and research issues. Med Sci Sports Exerc 2009; 41: $483-489$

10 Netopil B. Lymphödem bei Mammakarzinom. Frauenarzt 2008; 49 (4): $322-325$

11 Gesellschaft der epidemiologischen Krebsregister in Deutschland (GEKID). Krebs in Deutschland 2005/2006 Häufigkeiten und Trends. 7. Ausgabe; 2010: $56-59$

12 Zimmermann A, Szklarska A, Lipowicz A et al. Einfluss der manuellen Lymphdrainage auf die Schulterbeweglichkeit nach Brustkrebsoperation. Zeitschrift für Physiotherapeuten 2009; 7: 602-613

13 Szolnoky G, Lakatos B, Keskenny T et al. Intermittent pneumatic compression acts synergistically with manual lymphatic drainage in complex decongestive physiotherapy for breast cancer treatment-related lyymphedema. Lymphology 2009; 42: 188-194

14 Vignes S, Porcher S, Arrault $M$ et al. Long-term management of breast cancer-related lymphedema after intensive degongestive physiotherapy. Breast Cancer Res Treat 2007; 101: 285-290

15 Didem K, Ufuk YS, Serdar S et al. The comparison of two different physiotherapy methods in treatment of lymphedema after breast surgery. Breast Cancer Res Treat 2005; 93: 49-54

16 McNeely ML, Magee DJ, Lees AW. The addition of manual lymph drainage to compression therapy for breast cancer related lymphedema: a randomized controlled trial. Breast Cancer Res Treat 2004; 86: 95 - 106

17 Williams AF, Vadgama A, Franks PJ et al. A randomized controlled crossover study of manual lymphatic drainage therapy in women with breast cancer related lymphoedema. Eur J Cancer Care 2002; 11 : $254-261$

18 Sitzia J, Sobrido L, Harlow W. Manual lymphatic drainage compared with simple lymphatic drainage in the treatment of post mastectomy lymphoedema. Physiotherapy 2002; 88: 99-107

19 Szuba A, Cooke JP, Yousuf S et al. Decongestive lymphatic therapy for patients with cancer - related or primary lymphedema. Am J Med 2009; 109: $296-300$

20 Andersen L, Hojiris I, Erlandsen $M$ et al. Treatment of breast-cancerrelated lymphedema with or without manual lymphatic drainage. A randomized study. Acta Oncologica 2000; 39: 399-405
21 Dini D, Del Mastro L, Gozza A. The role of pneumatic compression in the treatment of post mastectomy lymphedema. A randomized phase II study. Ann Oncol 1998; 9: 187-190

22 Bertelli G, Venturini M, Forno $G$ et al. Conservative treatment of postmastectomy lymphedema: a controlled, randomized trial. Ann Oncol 1991; 2: $575-578$

23 Andersen L, Hojiris I, Erlandsen $M$ et al. Treatment of breast-cancerrelated lymphedema with or without manual lymphatic drainage. A randomized study. Acta Oncologica 2000; 39: 399-405

24 Beckermann M. Die Behandlung eines Lymphödems bei Brustkrebserkrankungen. In: GEK Heil-und Hilfsmittel-Report, Schriftenreihe zur Gesundheitsanalyse, Band 64, 2008: 49 - 74

25 Schiller-Frühwirth I. Manuelle Lymphdrainage beim primären und sekundären Lymphödem; Hauptverband der Österreichischen Sozialversicherungsträger HVB-EBM; 2007. EBM Berichte 2006: 1-23. www.hauptverband.at, Stand 07.12.2010

26 Deevogdt N, Van Kampen M, Geraerts I et al. Different physical treatment modalities for lymphoedema developing after axillary lymph node dissection for breast cancer: a review. Eur J Obstet Gynecol Reprod Biol 2010; 149: 3-9

27 Vignes S, Porcher S, Arrault $M$ et al. Long-term management of breast cancer-related lymphedema after intensive degongestive physiotherapy. Breast Cancer Res Treat 2007; 101: 285-290

28 Badger C, Preston N, Seers $K$ et al. Physical therapies for reducing and controlling lymphoedema of the limbs. Cochrane Database Syst Rev 2004; 4: CD003141

29 Johannson K, Albertsson M, Ingvar $C$ et al. Effects of compression bandaging with or without Manual Lymph Drainage Treatment in patients with postoperative arm lymphedema. Lymphology 1999; 32: $103-110$

30 Bertelli $G$, Venturini $M$, Forno $G$ et al. Conservative treatment of postmastectomy lymphedema: a controlled, randomized trial. Ann Oncol $1991 ; 2: 575-578$

31 Swedborg I. Effects of treatment with an elastic sleeve and intermittent pneumatic compression in post-mastectomy patients with lymphedema of the arm. Scand J Rehabil Med 1984; 16: 35-41

32 Schmitz KH, Ahmed RL, Troxel A et al. Weight lifting in women with breast-cancer-related lymphedema. N Engl Med 2009; 361: 664-673

33 Basen-Engquist K, Taylor CL, Rosenblum C et al. Randomized pilot test of a lifestyle physical activity intervention for breast cancer survivors. Patient Educ Couns 2006; 64: 225 - 234

34 Johannson $K$, Tibe $K$, Weibull A et al. Low intensity resistance exercise for breast cancer patients with arm lymphedema with or without compression sleeve. Lymphology 2005; 38: 167-180

35 McKenzie DC, Kalda AL. Effect of upper extremity exercise on secondary lymphedema in breast cancer patients: a pilot study. J Clin Oncol 2003; $21: 463-466$

36 Ahmed RL, Thomas W, Yee D et al. Randomized controlled trial of weight training and lymphedema in breast cancer survivors. J Clin Oncol 2006; 24: 2765-2772

37 Andersen L, Hojiris I, Erlandsen $M$ et al. Treatment of breast-cancerrelated lymphedema with or without manual lymphatic drainage. A randomized study. Acta Oncologica 2000; 39: 399-405

38 Beckermann M. Die Behandlung eines Lymphödems bei Brustkrebserkrankungen. In: GEK Heil-und Hilfsmittel-Report, Schriftenreihe zur Gesundheitsanalyse 2008; Band 64: 49-74

39 Kase K, Rock Stockheimer K, Piller N. Kinesio Taping for Lymphoedema and Chronic Swelling. Kenzo Kase Eigenverlag; 2006: 70

40 Kuhnke E. Volumenmessung aus Umfangbestimmung. Aus der Reihe Fol Angiologica XXIV.: 228-232

41 Deltombe T, Jamart J, Recloux S et al. Reliability and limits of agreement of circumferential, water displacement, and optoelectronic volumetry in the measurement of upper limb lymphedema. Lymphology 2007; 40: $26-34$

42 Seifart U, Kettner E, Albert U. Intertester-Variabilität eines „selfmade“Volumeters zur Bestimmung von Armvolumina. Eine prospektive Pilotstudie. Lymphologie in Forschung und Praxis 2010; 14: 14-19

43 Andersen L, Hojiris I, Erlandsen $M$ et al. Treatment of breast-cancerrelated lymphedema with or without manual lymphatic drainage. A randomized study. Acta Oncologica 2000; 39: 399-405

44 Meijer RS, Rietman JS, Geertzen JHB. Validity and Intra- and Interobserver reliability of an indirect volume measurement in patients with upper extremity lymphedema. Lymphology 2004; 37: 127-133

45 Karges JR, Mark BE, Stikeleather SJ. Concurrent validity of upper extremity volume estimates: Comparison of calculated volume derived 
from girth measurements and water displacement volume. Physical Therapy 2003; 83: 134-145

46 Bringezu G, Schreiner O. Lehrbuch der Entstauungstherapie. Heidelberg: Springer Medizin; 2010: 146-147

47 Siems W, Brenke R, Wiswedel I et al. Erhöhte Oxysterol-Spiegel beim chronischen Lymphödem. Lymphologie in Forschung und Praxis 2009; 13: $27-31$
48 Leduc $O$, Bourgeois P, Bastin R. Lymphatic resorption of proteins and pressotherapies. lème Congrès Groupe Européen de Lymphologie (G.E.L), Porto: 31.5.1985

49 Partsch H, Mostbeck A, Leitner G. Experimentelle Untersuchung zur Wirkung einer „Druckwellenmassage“ (Lymphapress) beim Lymphödem. Journal of Lymphology 1981 (1); Vol V: 35-39

50 Leduc A, Leduc 0 . Kombination verschiedener Therapiemaßnahmen in der Behandlung des Lymphödems. Zeitschrift für Lymphologie 1997; 1: $15-16$ 